\title{
Gems from the Work of Georgia Benkart
}

Tom Halverson and Arun Ram

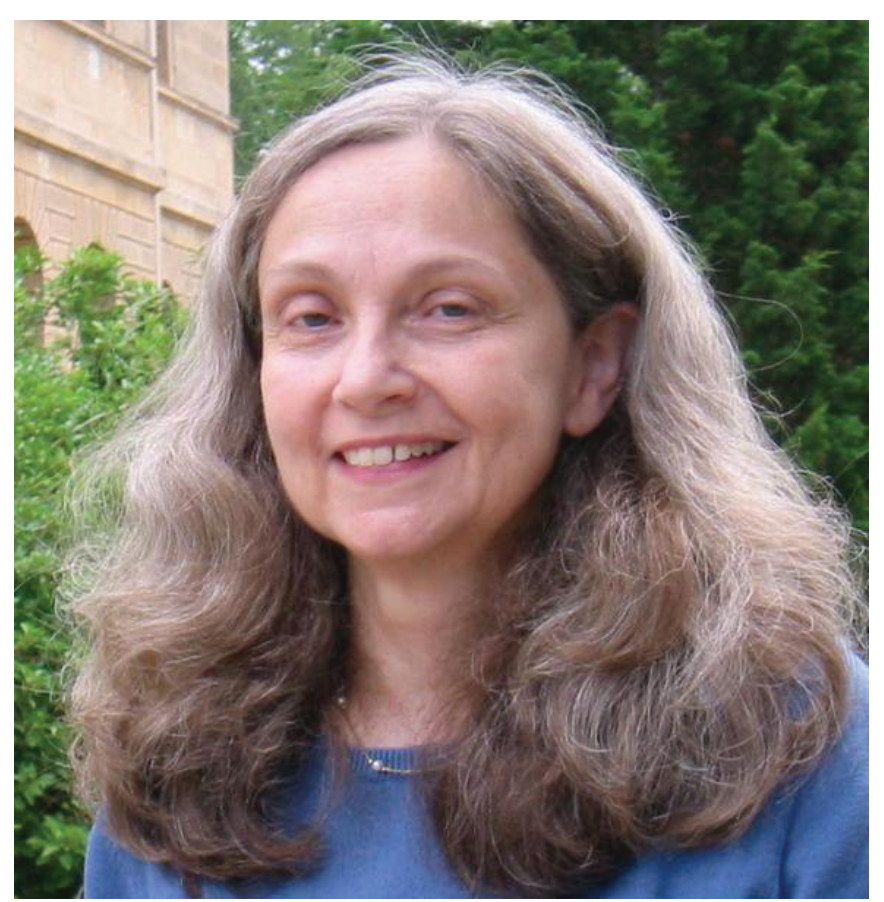

Georgia Benkart completed her PhD in 1974 at Yale University, where she was the 30th of Nathan Jacobson's 34 $\mathrm{PhD}$ students. From there she joined the faculty at the University of Wisconsin-Madison, where she is now Professor Emerita. Since her retirement from teaching she has provided tremendous service to the mathematical community, notably as President of the AWM and as an Associate Secretary of the AMS for more than a decade.

In this article we highlight a few selected gems from her extensive contribution to our field, organized in a roughly chronological sequence of vignettes and images (which can be read or viewed in any order). Our hope is that we can capture and transmit a snapshot of Georgia's rich

Tom Halverson is the Armstrong Professor of Mathematics at Macalester College. His email address is ha7verson@macalester. edu.

Arun Ram is a professor of mathematics at the University of Melbourne. His email address is aram@unime1b.edu.au.

Communicated by Notices Associate Editor Steven Sam.

For permission to reprint this article, please contact:

reprint-permission@ams.org.

DOI: https://doi.org/10.1090/noti2447 mathematics, beautiful style, and wonderful mathematical personality.

Classifying simple Lie algebras. In algebra in 1974, the air was thick with the classification of finite simple groups, with new finite simple groups being discovered in a frenzy, and the question always in the air:

\section{"Have we found them all?"}

At that time there was another such classification effort beginning: a search for all of the finite-dimensional simple Lie algebras.

In characteristic 0 the problem had been completed by Cartan and Killing around 1894, resulting in the list of Dynkin diagrams (Figure 1), which are in bijection with the finite-dimensional simple Lie algebras. Over an algebraically closed field of characteristic $p>7$, four additional series occur:

- the Witt Lie algebras $W(m, \underline{n})$,

- the special Lie algebras $S(m, \underline{n})^{(1)}$,

- the Hamiltonian Lie algebras $H(2 m, \underline{n})^{(2)}$,

- the contact Lie algebras $K(2 m+1, \underline{n})^{(1)}$.

The monograph by Benkart, Gregory, and Premet [BGP09] provides complete details on these algebras. They are known as the generalized Cartan-type Lie algebras, because they are derived from Cartan's four infinite families (Witt, special, Hamiltonian, contact) of infinitedimensional complex Lie algebras. Cartan's work set the stage for Kostrikin-Šafarevič [KŠ66], who identified the above four unifying families of simple Lie algebras living in the Witt algebras. Earlier work of George Seligman [Sel67] (also at Yale) emphasized the role and the importance of the Lie algebras of Cartan type. George was one of Jacobson's first students and Georgia was one of his last.

In 1966, Kostrikin and Šafarevič conjectured that the Cartan-type Lie algebras and the Lie algebras coming from characteristic 0 were all of the finite-dimensional simple Lie algebras (over an algebraically closed field) in characteristic $p$. The original formulation was for "restricted" Lie algebras, and the general statement for finite-dimensional simple Lie algebras is the "Generalized Kostrikin-Šafarevič conjecture." 


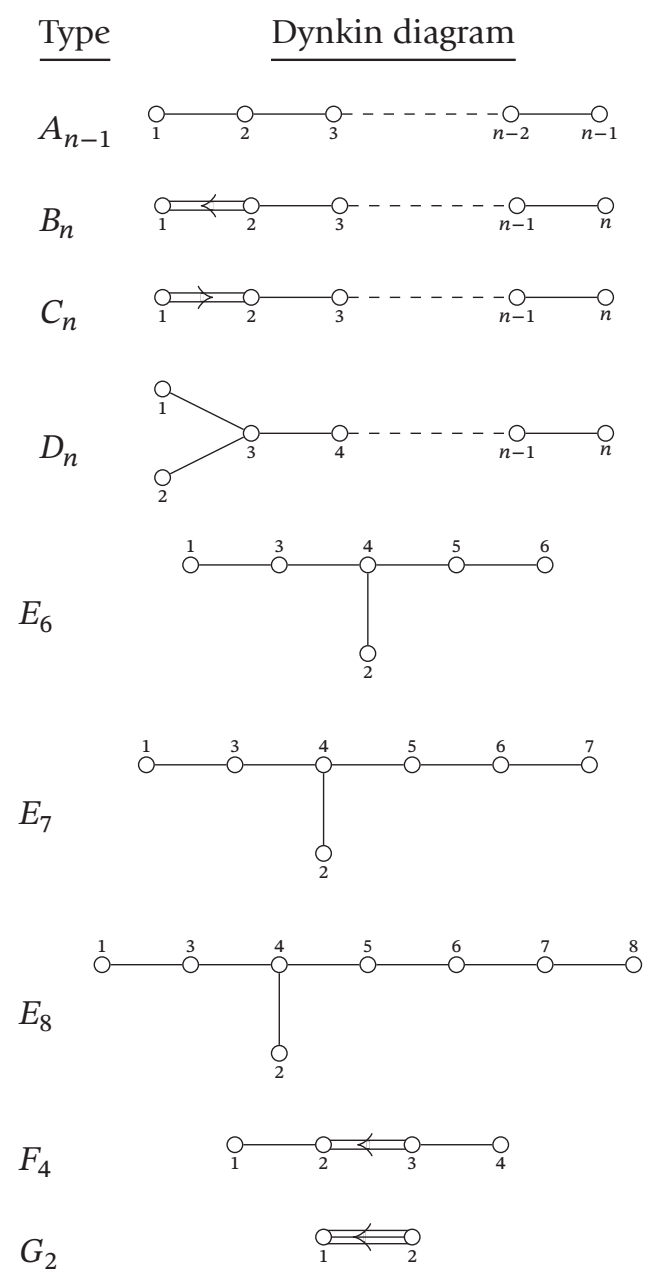

Figure 1. The classification of finite-dimensional simple Lie algebras in characteristic 0 is by the above Dynkin diagrams. In characteristic $p>3$ there are five additional series of algebras:

(1) the Witt Lie algebras $W(m, \underline{n})$,

(2) the special Lie algebras $S(m, \underline{n})^{(1)}$,

(3) the Hamiltonian Lie algebras $H(2 m, n)^{(2)}$, and

(4) the contact Lie algebras $K(2 m+1, \underline{n})^{(1)}$;

and when $p=5$ there is one more additional series:

(5) the Melikyan Lie algebras $M(2, \underline{n})$.

The study and proof of the Kostrikin-Šafarevič conjecture inspired work by many people around the world. Georgia brought to Wisconsin the mindset of the Jacobson school, emphasizing a module-theoretic approach to the classification of algebraic systems. She joined a thriving algebra community that included Marty Isaacs, Marshall Osborn, Donald Passman, and Louis Solomon. Also in the thick of the action around the Kostrikin-Šafarevič conjecture were Richard Block, Robert Wilson (who had been a student of George Seligman at Yale), and Victor Kac, who seemed to be everywhere, classifying all things Lie.

Benkart and Osborn [BO84] classified the finitedimensional simple Lie algebras of characteristic $p>7$ with a one-dimensional Cartan subalgebra, showing that they are either $\mathfrak{g l}(2)$ or Albert-Zassenhaus Lie algebras (the algebras $W(1, \underline{n})$ and a family of Hamiltonian Lie algebras). Their paper [BO90] studied the subalgebra $L^{(\alpha)}=$ $L_{0} \oplus L_{\alpha} \oplus L_{2 \alpha} \oplus \cdots \oplus L_{(p-1) \alpha}$ of a finite-dimensional simple Lie algebra $L$ determined by a root $\alpha$. Modulo the radical, these one-sections $L^{(\alpha)}$ are isomorphic to either $\mathfrak{s} \mathfrak{l}(2)$, $W(1, \underline{1})$, or to a subalgebra of $H(2, \underline{1})$ containing $H(2, \underline{1})^{(2)}$.

The results of Benkart and Osborn, along with their proof techniques, were ultimately absorbed into the general classification process. In the 1990s, Alexander Premet and Helmut Strade pulled it all together, methodically completing every step to a full classification.

Of course, as with any huge project, there were many other important contributors in addition to those named here. In the middle of it all, in 1980, Melikyan found a new finite-dimensional simple Lie algebra in characteristic 5 , of dimension 125 . That certainly put a wrench into things, and increased the worry that, in those small $p$ cases, there might exist even more fascinating and untamed algebras that nobody had seen before. Fortunately, now the whole project is finished for $p>3$ and is comprehensively exposited in the 1100 pages of the three volumes of Helmut Strade's books, Simple Lie algebras over fields of positive characteristic Vols. I, II, and III [Str17a, Str17b, Str13].

Quoting from the Math Review of Vol. III:

Kac's recognition theorem is one major result whose proof is not included in the book. All details for an arbitrary $p>3$ can be found in a paper of G.M. Benkart, T.B. Gregory and Premet [BGP09].

The Recognition Theorem was a hugely important step on the long road to completion of the classification. To quote from the introduction of [BGP09]: "The Recognition Theorem is used several times throughout the classification; its first application results in a complete list of the simple Lie algebras of absolute toral rank two, and its last application yields a crucial characterization of the Melikyan Lie algebras, thereby completing the classification." Finally those mysterious Melikyan algebras (they had multiplied in the interim and become a whole family) were under control in the sense that the freedom that causes them to appear had been pinpointed, and it had been checked carefully that this freedom doesn't cause other sporadic examples of this nature. The monograph of Benkart, Gregory, and Premet is a wonderful work to read: thorough, efficient, elementary, with precise definitions; it contains a clear big-picture point of view. It is absolutely beautifully written.

Infinite dimensions and magic squares. The structure of a finite-dimensional Lie algebra $\mathfrak{g}$ corresponding to one of the Dynkin diagrams in Figure 1 is governed by its root 


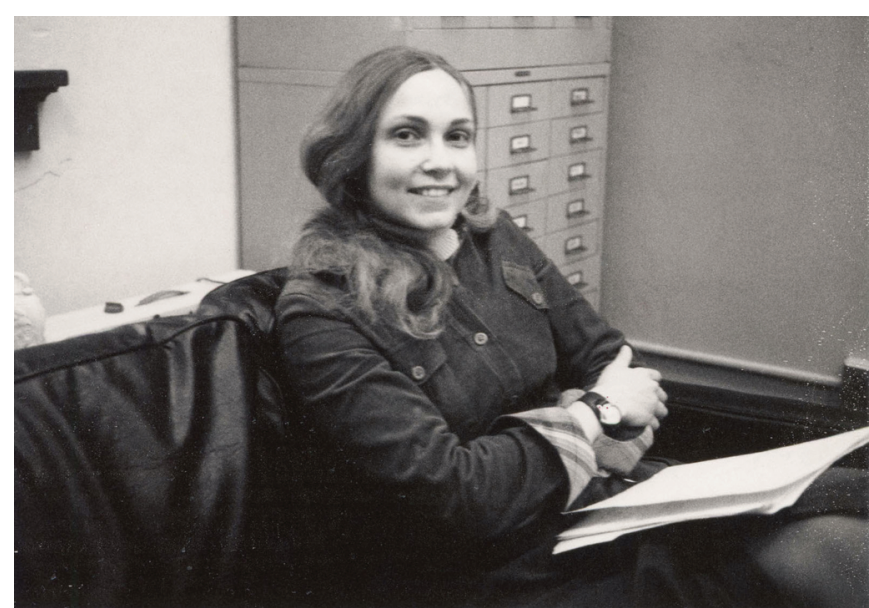

Figure 2. Georgia Benkart on February 22, 1979 during a visit to Indiana University. An image from the Paul R. Halmos Photograph Collection.

system $\Delta$, and $\mathfrak{g}$ decomposes into a direct sum of the form,

$$
\mathfrak{g}=\mathfrak{h} \oplus\left(\bigoplus_{\alpha \in \Delta} \mathfrak{g}_{\alpha}\right), \quad \text { with } \quad \operatorname{dim}\left(\mathfrak{g}_{\alpha}\right)=1
$$

and $\operatorname{dim}(\mathfrak{h})$ equal to the number of vertices in the Dynkin diagram. Furthermore, the root system $\Delta$ has a geometric description connecting it to the world of polytopes (see Figure 3).

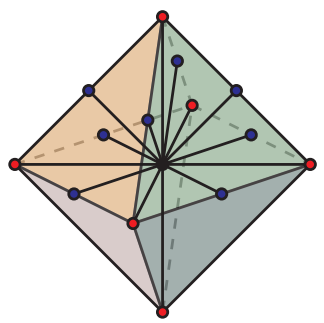

The blue and red vectors form the root system $\Delta$ of the finite-dimensional Lie algebra $\mathfrak{g}=\mathfrak{s p}_{6}$.

Figure 3. The root system $\Delta$ for a Lie algebra $g$ corresponding to the Dynkin diagram $C_{3}$. The root system $\Delta$ consists of the vectors from the center to the vertices and from the center to the midpoints of the edges of the octahedron. See equation (1).

The second half of the 20th century produced a huge expansion into the universe of infinite-dimensional Lie algebras. The finite-dimensional Cartan-type Lie algebras in Figure 1 are the characteristic $p$ versions of infinitedimensional characteristic 0 Lie algebras that arose from Cartan's study of "pseudogroups." The study of Feynman path integrals and the development of string theory also produced new examples of infinite-dimensional Lie algebras with interesting structure.

The underlying structure of the infinite-dimensional Lie algebra $L$ comes from a finite-dimensional $\mathfrak{g}$ sitting inside $L$. This property was formalized in the early 1990 s by Berman and Moody when they defined $\Delta$-graded Lie algebras. A $\Delta$-graded Lie algebra $L$ contains a subalgebra $\mathfrak{g}$ corresponding to a Dynkin diagram, and the whole Lie algebra $L$ decomposes into root spaces $L_{\mu}$ indexed by the root system $\Delta$ of $\mathfrak{g}$,

$$
\mathfrak{g} \subseteq L \quad \text { and } \quad L=\bigoplus_{\alpha \in \Delta \cup\{0\}} L_{\alpha} .
$$

Berman and Moody classified the $\Delta$-graded Lie algebras for which the Dynkin diagram does not have double or triple edges by viewing them as Lie algebras analogous to $\mathfrak{S l}_{n}(R)$, where $R$ is an (associative) algebra. Favorite examples are the polynomial rings $R=\mathbb{C}\left[t_{1}, \ldots, t_{n}\right]$ and the Laurent polynomial rings $R=\mathbb{C}\left[t_{1}^{ \pm 1}, \ldots, t_{n}^{ \pm 1}\right]$, but $R$ can be much more general.

Berman and Moody's classification leads one to wonder what happens when the Dynkin diagram has multiple edges. Efim Zelmanov had started to study these cases, and in the course of his work gave a few lectures in the seminar at the University of Wisconsin. One morning Georgia came in and indicated that she thought that some of the ideas from her thesis might apply to this question. It didn't take long before Georgia and Efim hunkered down and quickly polished off all the other cases and completed the amazing theorem that

$$
\begin{aligned}
& \text { all } \Delta \text {-graded Lie algebras have the form } \\
& L=(\mathfrak{g} \otimes A) \oplus(W \otimes B) \oplus D,
\end{aligned}
$$

where $\mathfrak{g}$ is a finite-dimensional Lie algebra with root system $\Delta, W$ is a small $\mathfrak{g}$-module, and $D$ is a subalgebra of derivations that acts on the algebra $\mathfrak{a}=A \oplus B$. Hence the infinite-dimensional Lie algebra $L$ is something like that in Figure 4, where $A$ and $B$ are visualized as appendages to the root system $\Delta$. The Benkart-Zelmanov paper [BZ96] explaining how this works has become a classic.

Georgia didn't stop there. There are two basic steps in the classification of $\Delta$-graded Lie algebras:

First: One has to show that the only possible forms that a $\Delta$-graded Lie algebra can take are $L=(\mathfrak{g} \otimes A) \oplus$ $(W \otimes B) \oplus D$.

Second: After narrowing down the possibilities, one has to show that they all occur in reality and do, in fact, produce $\Delta$-graded Lie algebras.

This second step is obtained by powerful constructions which go by various names (see Tables 1 and 2): "Freudenthal's magic square," the "Tits-Kantor-Koecher construction," "generalized octonions." These constructions were originally conceived to build the Lie algebras corresponding to the Dynkin diagrams $E_{6}, E_{7}, E_{8}, F_{4}$, and $G_{2}$. They were vastly generalized by Benkart-Zelmanov to construct $\Delta$-graded Lie algebras and by Benkart-Elduque and Elduque to extend to exceptional Lie superalgebras and Lie algebras and Lie superalgebras in characteristic $p$. 


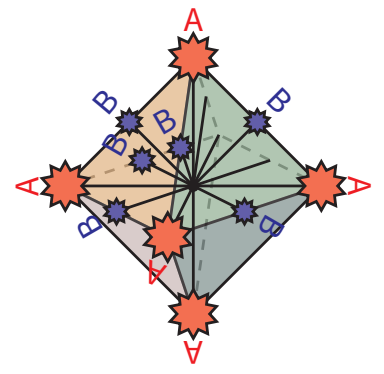

Figure 4. The infinite-dimensional $\Delta$-graded Lie algebra corresponding to the root system $\Delta$ for the Dynkin diagram $C_{3}$. The octahedron provides the structure of the root system $\Delta$ of the finite-dimensional Lie algebra $g$. The $\Delta$-graded Lie algebra $L=(\mathfrak{g} \otimes A) \oplus(W \otimes B) \oplus D$ is built by fitting $\mathfrak{g}$-modules $A$ and $B$ into sockets on the mother board $g$ labeled by the elements of $\Delta$. See (2).

\begin{tabular}{c|cccc}
$\mathcal{T}(C, J)$ & $H_{3}(\mathbb{F})$ & $H_{3}\left(\mathbb{F}^{2}\right)$ & $H_{3}\left(M_{2}(\mathbb{F})\right)$ & $H_{3}(C(\mathbb{F}))$ \\
\hline $\mathbb{F}$ & $A_{1}$ & $A_{2}$ & $C_{3}$ & $F_{4}$ \\
$\mathbb{F}^{2}$ & $A_{2}$ & $A_{2} \oplus A_{2}$ & $A_{5}$ & $E_{6}$ \\
$M_{2}(\mathbb{F})$ & $C_{3}$ & $A_{3}$ & $D_{6}$ & $E_{7}$ \\
$C(\mathbb{F})$ & $F_{4}$ & $E_{6}$ & $E_{7}$ & $E_{8}$
\end{tabular}

Table 1. The Tits-Kantor-Koecher construction. In this table $\mathbb{F}^{2}=\mathbb{F} \times \mathbb{F}, M_{2}(\mathbb{F})$ denotes the algebra of $2 \times 2$ matrices with entries from a field $\mathbb{F}$, and $H_{3}\left(C^{\prime}\right)$ is the Jordan algebra of $3 \times 3$ Hermitian matrices over the unital composition algebra $C^{\prime}$.

\begin{tabular}{c|cccc}
$\mathfrak{g}\left(C, C^{\prime}\right)$ & 1 & 2 & 4 & 8 \\
\hline 1 & $A_{1}$ & $A_{2}$ & $C_{3}$ & $F_{4}$ \\
2 & $A_{2}$ & $A_{2} \oplus A_{2}$ & $A_{5}$ & $E_{6}$ \\
4 & $C_{3}$ & $A_{3}$ & $D_{6}$ & $E_{7}$ \\
8 & $F_{4}$ & $E_{6}$ & $E_{7}$ & $E_{8}$
\end{tabular}

Table 2. Freudenthal's magic square or the symmetric (Vinberg) construction. The rows are indexed by $\operatorname{dim}(C)$, and the columns are indexed by $\operatorname{dim}\left(C^{\prime}\right)$.

The construction has two forms: the first method is to take a Jordan algebra $J$ and a composition algebra $C$ and twist them together to get a Lie algebra $L=\mathcal{T}(C, J)$. The other version of the construction (introduced by Vinberg) builds the Lie algebra $L$ from two composition algebras $C$ and $C^{\prime}$. In this version, the symmetry of Freudenthal's magic square is embedded into the construction.

The wonderful article of Elduque in the Tits 80th birthday volume [Eld11] provides an accessible survey of the various constructions of Freudenthal's magic square, along with recent advances in the theory involving Georgia and her coauthors and a nice entrée into open questions and current research in this vein. The original paper of Benkart-Zelmanov [BZ96] classified $\Delta$-graded Lie algebras for the cases where the Dynkin diagram of $\Delta$ is $B_{r}, C_{r}, F_{4}$, and $G_{2}$. The AMS Memoir of Allison, Benkart, and Gao [ABG02] provides an amazing resource for understanding all parts of the classification of $\Delta$-graded Lie algebras, the analysis of their derivations, central extensions and invariant forms, and their constructions, including the TitsKantor-Koecher constructions.

Elemental Lie algebras. Imagine that it is the early 1800 s and you are Dalton, or Gay-Lussac, or Avogadro, trying to figure out how atoms combine to make molecules. There are two fundamental problems to solve:

(a) What are the individual elements?

(b) How do they combine to make molecules?

Now imagine that it is the turn of the 21st century and you are Georgia Benkart trying to figure out how Lie algebras are built. There are two fundamental problems:

(a) What are the littlest Lie algebras?

(b) How do they combine to make larger Lie algebras?

A motivating phenomenon is that all finite-dimensional simple Lie algebras $\mathfrak{g}$ (in characteristic 0) and all KacMoody Lie algebras are constructed from the little Lie algebras $\mathfrak{S l}_{2}$ glued together appropriately.

Letting $[a, b]=a b-b a$,

$$
\mathfrak{s l}_{2}=\left\{\left(\begin{array}{cc}
a_{1} & a_{2} \\
a_{3} & -a_{1}
\end{array}\right)\right\}=\operatorname{span}\{x, y, h\},
$$

where

$$
x=\left(\begin{array}{ll}
0 & 1 \\
0 & 0
\end{array}\right), \quad y=\left(\begin{array}{ll}
0 & 0 \\
1 & 0
\end{array}\right), \quad h=\left(\begin{array}{cc}
1 & 0 \\
0 & -1
\end{array}\right),
$$

and

$$
[x, y]=h, \quad[h, x]=2 x, \quad[h, y]=-2 y .
$$

Another little Lie algebra is the three-dimensional Heisenberg Lie algebra

$$
\mathcal{H}=\left\{\left(\begin{array}{ccc}
0 & a_{1} & a_{3} \\
0 & 0 & a_{2} \\
0 & 0 & 0
\end{array}\right)\right\}=\operatorname{span}\{x, y, h\},
$$

where

$$
\begin{gathered}
x=\left(\begin{array}{lll}
0 & 1 & 0 \\
0 & 0 & 0 \\
0 & 0 & 0
\end{array}\right), \quad y=\left(\begin{array}{lll}
0 & 0 & 0 \\
0 & 0 & 1 \\
0 & 0 & 0
\end{array}\right), \\
h=\left(\begin{array}{lll}
0 & 0 & 1 \\
0 & 0 & 0 \\
0 & 0 & 0
\end{array}\right),
\end{gathered}
$$

and

$$
[x, y]=h, \quad[h, x]=0, \quad[h, y]=0 .
$$

These algebras are strikingly similar in presentation, but different in application. If one has these examples in mind, then it is not very surprising that Georgia has sequences of papers engaged in the study of families of "elemental" algebras over a field $\mathbb{F}$ : 
(A) the parametric family

$A_{h}$, generated by $x$ and $y$ with

$$
[x, y]=h \text {, where } h \in \mathbb{F}[x] \text {, and }
$$

(B) the down-up algebras [BR98], depending on parameters $\alpha, \beta, \gamma \in \mathbb{F}$ :

$$
\begin{gathered}
A(\alpha, \beta, \gamma) \text {, generated by } u \text { and } d \text { with } \\
d^{2} u=\alpha d u d+\beta u d^{2}+\gamma d \text { and } \\
d u^{2}=\alpha u d u+\beta u^{2} d+\gamma u .
\end{gathered}
$$

If $\gamma=0, \beta=-1$, and $\alpha=2$, then $d^{2} u-2 d u d+u^{2} d=0$, and we recover the Heisenberg algebra. This is because in $\mathcal{H}$, the relation $0=[h, x]$ expands to $0=h x-x h=$ $[x, y] x-x[x, y]=(x y-y x) x-x(x y-y x)=x y x-y x^{2}-$ $x^{2} y+x y x=-\left(x^{2} y-2 x y x+y x^{2}\right)$.
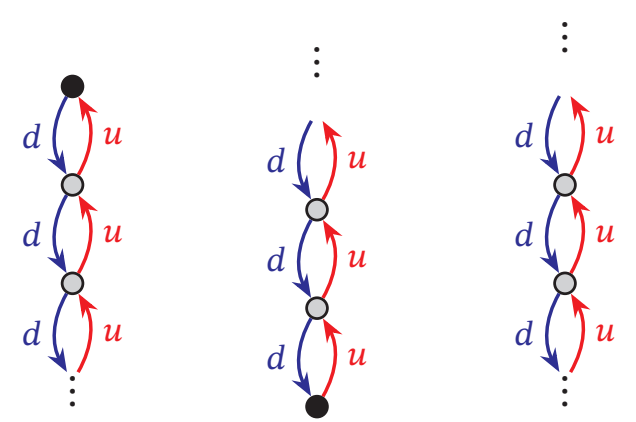

highest weight lowest weight doubly infinite

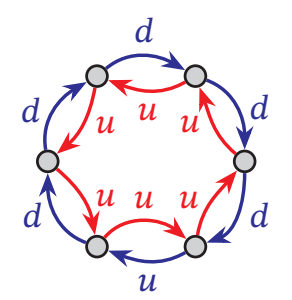

finite-dimensional

Figure 5. Irreducible modules for the down-up algebras $A(\alpha, \beta, \gamma)$. Up to constants depending on the parameters $\alpha, \beta, \gamma$, the $u$ operators act according the red edges and the $d$ operators act according the blue edges. The black vertex represents the highest weight and the lowest weight, respectively. See (4).

These algebras $A_{h}$ and $A(\alpha, \beta, \gamma)$ capture the core underlying structures that join together to make larger Lie algebras and their quantum groups. Georgia and her collaborators have done thorough studies of the properties of these "little quantum groups" by determining all of the following: automorphisms, inner automorphisms, centers, derivations, inner derivations, their Hochschild cohomology $H H^{1}$, prime ideals, primitive ideals, Duflo correspondences between primitive ideals and annihilators of simple modules, highest weight modules, lowest weight modules, finite-dimensional modules, Whittaker modules, and also some tensor product rules for simple modules in case that wasn't enough already.

Just to highlight a tiny portion of these results, Georgia and her collaborators determine precisely all the possible "shapes" of irreducible modules of down-up algebras $A(\alpha, \beta, \gamma)$. These are shown pictorially in Figure 5 .

Because the algebras $A_{h}$ and $A(\alpha, \beta, \gamma)$ are so "elemental" (generalizing the structures from $\mathfrak{s l}_{2}$ and threedimensional Hesenberg algebras), one has confidence that they will be useful to mathematicians of the future in the same way that intimate knowledge of Mendeleev's periodic table is indispensible for any post-19th century chemist. The elemental Lie algebras are the atoms from which larger Lie algebras and quantum groups that arise in nature (i.e., many other parts of mathematics and physics) are built.

Talking the talk: A Tale of Two Groups. In a Dickensian plenary address at the 1994 Joint Math Meetings, Georgia told the story of Schur-Weyl duality as a "Tale of Two Groups." See [Ben96]. The protagonist is a group $G$ acting on tensor powers of a defining representation, and the antagonist is the algebra of endomorphisms $\operatorname{End}_{G}\left(V^{\otimes n}\right)$ that commute with $G$. See Figure 6 .

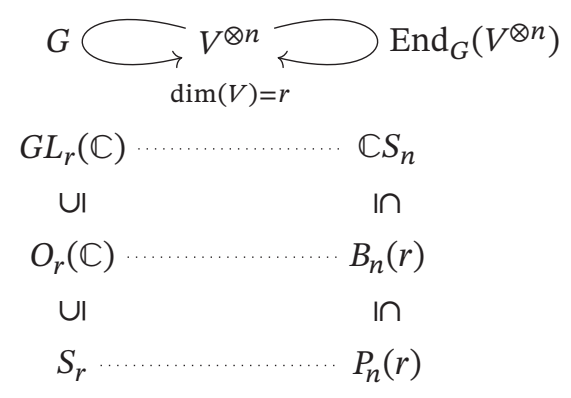

Figure 6. Schur-Weyl duality between the general linear group $G L_{r}(\mathbb{C})$ and the symmetric group $S_{n}$, between the orthogonal group $O_{r}(\mathbb{C})$ and the Brauer algebra $B_{n}(r)$, and between the symmetric group $S_{n}$ and the partition algebra $P_{n}(r)$.

In his groundbreaking thesis at the turn of the 20th century, Schur used these methods to construct the irreducible polynomial representations of the general linear group $G=G L_{r}(\mathbb{C})$. He showed that $\operatorname{End}_{G}\left(V^{\otimes n}\right)$ is generated by $\mathbb{C} S_{n}$, the algebra of permutations, displayed here as a permutation diagram,

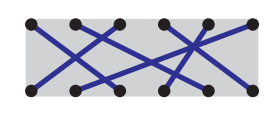
acting on $V^{\otimes n}$ by tensor place permutation.

In the 1930s Brauer showed that if $G=O_{n}(\mathbb{C})$, then $\operatorname{End}_{G}\left(V^{\otimes n}\right)$ is generated by the algebra of Brauer diagrams, 
which correspond to arbitrary matchings of $2 n$ vertices,

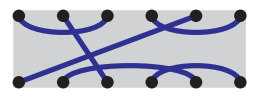

acting on $V^{\otimes n}$ by permutation and contraction onto subspaces.

In about 1990, Paul Martin and Vaughan Jones showed that if $G=S_{r}$, the symmetric group, then the centralizer $\operatorname{End}_{G}\left(V^{\otimes n}\right)$ is generated by set partition diagrams,

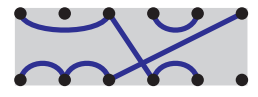

acting on $V^{\otimes n}$ by permutation, contraction, and fragmentation.

Set partition diagrams multiply with one another via concatenation:

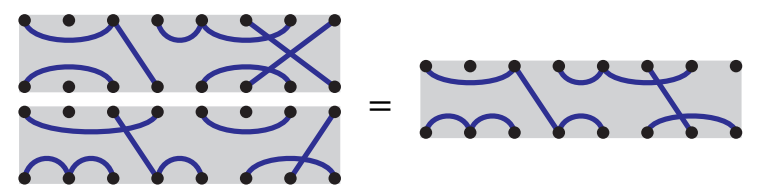

Schur-Weyl duality allows information to flow back and forth between the group $G$ and its centralizer $\operatorname{End}_{G}\left(V^{\otimes n}\right)$. In an AMS Memoir [BBL90] Georgia and her coauthors, Dan Britten and Frank Lemire, study finitedimensional representations of $G L_{r}(\mathbb{C}), S L_{r}(\mathbb{C}), O_{r}(\mathbb{C})$, and $S p_{2 r}(\mathbb{C})$. They identify submodules for these $G$ inside the tensor space $V^{\otimes n}$ and use the combinatorics of the centralizer, for example,

\begin{tabular}{l|l|l|l|l|l|}
\hline 1 & 1 & 2 & 3 & 3 \\
\hline 2 & 4 & 4 & 5 & \\
\hline 3 & 5 & 5 & 7 & \\
\hline 4 & 7 & 7 & \\
\hline 5 & 9 & & \\
Coung tableaux & &
\end{tabular}

to understand stability properties for irreducible $G$ modules as $r$ grows. Georgia [Ben90] and Sheila Sundaram [Sun90] each give elegant descriptions of these combinatorial methods in representation theory.

In 1989, in a collaboration $\left[\mathrm{BCH}^{+} 94\right]$ with five graduate students at the University of Wisconsin, Georgia defined the walled-Brauer algebra by determining the centralizer of the $G L_{n}(\mathbb{C})$ on $V^{\otimes n} \otimes\left(V^{*}\right)^{\otimes m}$, where $V^{*}$ is the dual module to $V$. This time, the diagrams come with a left part and a right part separated by a wall, with the constraint that horizontal edges must cross the wall and top-to-bottom edges must not cross the wall,

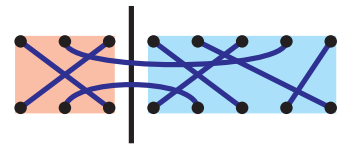

acting on $V^{\otimes n} \otimes\left(V^{*}\right)^{\otimes m}$.
This collaboration, with Georgia leading a group of five junior mathematicians at once, was unusual at the time. Now, this is more common and one finds, among Georgia's recent papers, several team collaborations that include early-career researchers who have been stimulated by Georgia's leadership. Not only is Georgia a natural and inspiring mentor for these teams, but she initiated them long before there were organizations like Banff (see Figure 11) and MSRI helping so effectively to make it happen.

Walking the walk: The Representation Theory Way. In 2014, Georgia delivered the Noether Lecture at the International Congress of Mathematicians in Seoul, Korea entitled, "Walking on Graphs the Representation Theory Way." The motivating idea is that one can build

every irreducible $G$-module $V_{i}$ from a single well-chosen $G$-module $V$,

by applying idempotents $p_{i}$ of $\operatorname{End}_{G}\left(V^{\otimes n}\right)$ to $V^{\otimes n}$. The idempotent

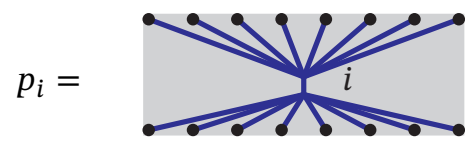

is a projection onto the irreducible $G$ summand $V_{i}$. A powerful way to study this is by building a graph that keeps track of what happens when one tensors by $V$. This representation graph, or McKay quiver, has vertices $V_{i}$ and $r$ edges $V_{i} \rightarrow V_{j}$ if $V_{j}$ appears $r$ times in $V_{i} \otimes V$. For example, if $G=\left\{1, g, g^{2}, \ldots, g^{n-1}\right\}$ is the cyclic group of order $n$, and $V$ is the two-dimensional representation of $G$ corresponding to the matrix

$$
g=\left(\begin{array}{cc}
\omega^{-1} & 0 \\
0 & \omega
\end{array}\right), \quad \omega=e^{2 \pi i / n},
$$

then the representation graph of the pair $(G, V)$ is

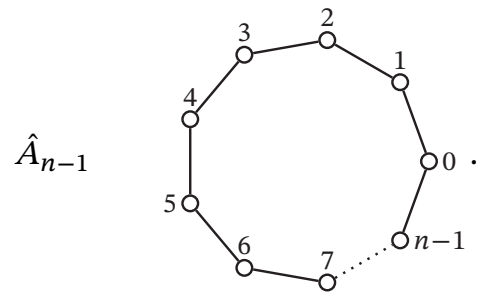

If $G=\left\{g, h \mid g^{2 n}=1, h^{2}=g^{n}, g h=h g^{-1}\right\}$ is the binary dihedral group of order $4 n$ and $V$ is the two-dimensional representation given by the matrices

$$
g=\left(\begin{array}{cc}
\zeta^{-1} & 0 \\
0 & \zeta
\end{array}\right), \zeta=e^{\pi i / n}, \quad \text { and } \quad h=\left(\begin{array}{cc}
0 & i \\
i & 0
\end{array}\right),
$$

then the representation graph of the pair $(G, V)$ is

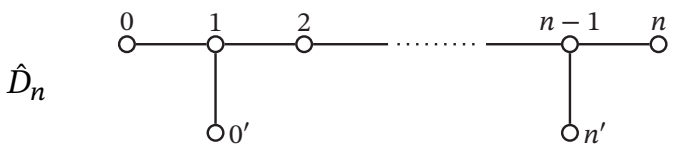


Finally, if $G$ is one of the three polyhedral groups,

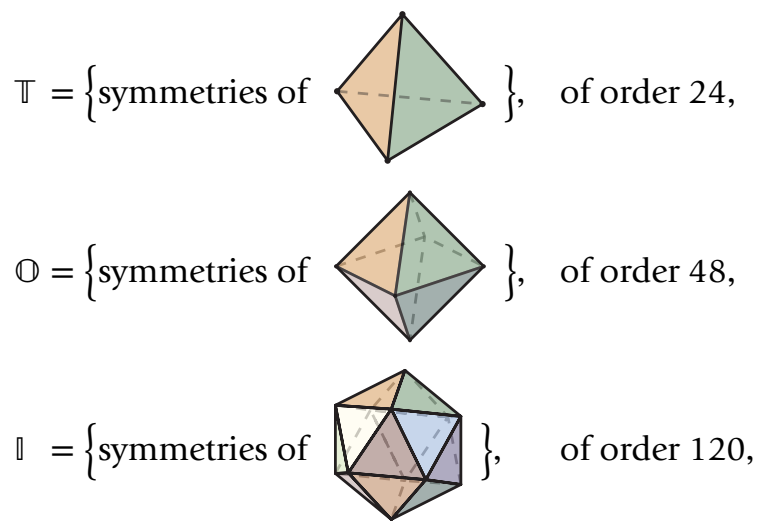

and $V$ is the two-dimensional representation of $G$, then the representation graphs of the pairs $(G, V)$ are the graphs in Figure 7. The observation that the graphs $\hat{A}_{n-1}, \hat{D}_{n}, \hat{E}_{6}, \hat{E}_{7}$, $\hat{E}_{8}$ are exactly the "simply-laced affine Dynkin diagrams" is the amazing McKay correspondence. These same graphs also describe (see [Kac90] and [Bri71]) the internal structure of the Lie algebras of loop groups as well as the structure of the subregular nilpotent orbits for reductive algebraic groups!

In these examples, if we now let $Z_{n}=\operatorname{End}_{G}\left(V^{\otimes n}\right)$, then the two commuting actions of $G$ and $Z_{n}$ on $V^{\otimes n}$

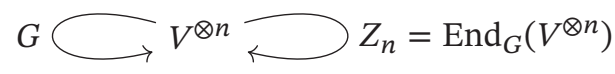

give a decomposition of $V^{\otimes k}$ into irreducible $\left(G, Z_{n}\right)$ bimodules,

$$
V^{\otimes n}=\bigoplus_{i} V_{i} \otimes Z_{n}^{i}
$$

The walks on the representation graph $\Gamma(G, V)$ encode multiplicities and dimensions:

$$
\begin{aligned}
& \#\{\text { walks of length } n \text { from } 0 \text { to } i \text { on } \Gamma(G, V)\} \\
& \quad=\text { multiplicity of the } G \text {-module } V_{i} \text { in } V^{\otimes n} \\
& \quad=\text { dimension of the } Z_{n} \text {-module } Z_{n}^{i}
\end{aligned}
$$

and $\operatorname{dim}\left(Z_{n}\right)$ is the number of walks that come back home (to the node labeled 0 ) after $2 n$ steps:

$$
\begin{aligned}
& \operatorname{dim}\left(Z_{n}\right)=\sum_{i} \operatorname{dim}\left(Z_{n}^{i}\right)^{2} \\
& =\#\{\text { walks of length } 2 n \text { from } 0 \text { to } 0 \text { on } \Gamma(G, V)\} .
\end{aligned}
$$

A particularly elegant way to enumerate walks on the representation graph $\Gamma(G, V)$ is to expand them into paths on the corresponding Bratteli diagram $\mathcal{B}(G, V)$, which is an infinite lattice organized so that the nodes on level $n$ are those that can be reached by an $n$-step walk starting at the root on $\Gamma(G, V)$ (see Figure 8).

With several collaborators, Georgia has used walks on these representation graphs to answer many questions in combinatorial representation theory. To name just a
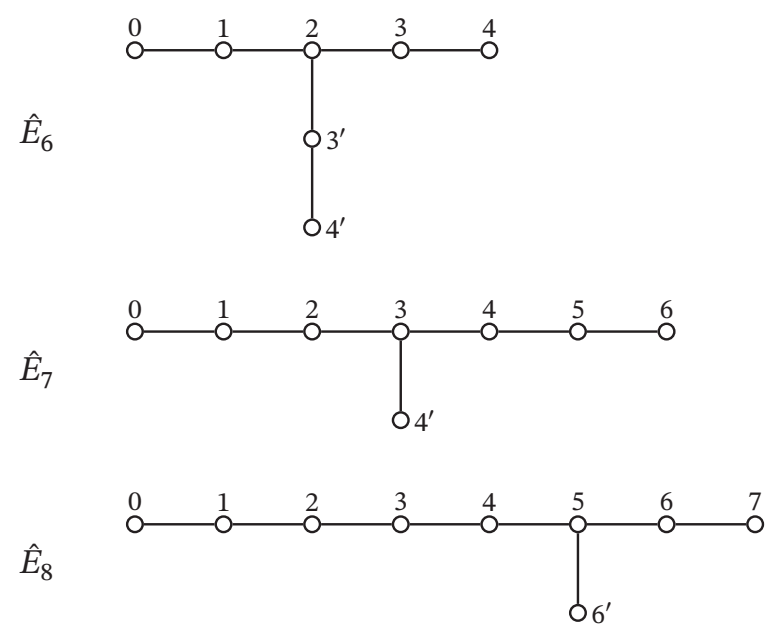

Figure 7. The representation graphs of the binary tetrahedral, octahedral, and icosahedral groups are the simply-laced affine Dynkin diagrams of type $\hat{E}_{6}, \hat{E}_{7}$, and $\hat{E}_{8}$.

few: they describe the projection operators in McKay and Motzkin centralizer algebras; they characterize the kernel of the partition algebra on tensor space; they describe walks on hypercubes; and they are used to perform chip firing on Dynkin diagrams and McKay quivers.

Fusion rules! Georgia's most recent talks and collaborations have centered around fusion rules. Fusion matrices encode the rules that determine the decomposition of the tensor product of two modules into a direct sum of simple modules. In the case of the McKay correspondence, the fusion matrices are the adjacency matrices of the representation graphs in Figure 7, and in conformal field theory in physics, integrable models are described by the fusion rules for their charges.

In a group project $\left[\mathrm{BBK}^{+} 21\right]$ that began at the workshop in Leeds for Women in Noncommutative Algebra and Representation Theory (WINART3), Georgia and her collaborators compute fusion matrices for certain classes of finitedimensional Hopf algebras. They express the eigenvalues and eigenvectors of these matrices in terms of Chebyshev polynomials, furthering the case that Chebyshev polynomials are as dense in representation theory as they are in numerical analysis. A key step is to relate the eigenvectors to characters, and an overarching question in this work is to find a good notion of a character table for a Hopf algebra.

In another exciting collaboration, Georgia worked with Persi Diaconis, Martin Liebeck, and Pham Huu Tiep (see [BDLT20]) at MSRI to use fusion matrices to analyze families of Markov chains. They studied walks in a similar manner to the case pictured in Figure 8 above, except now using 


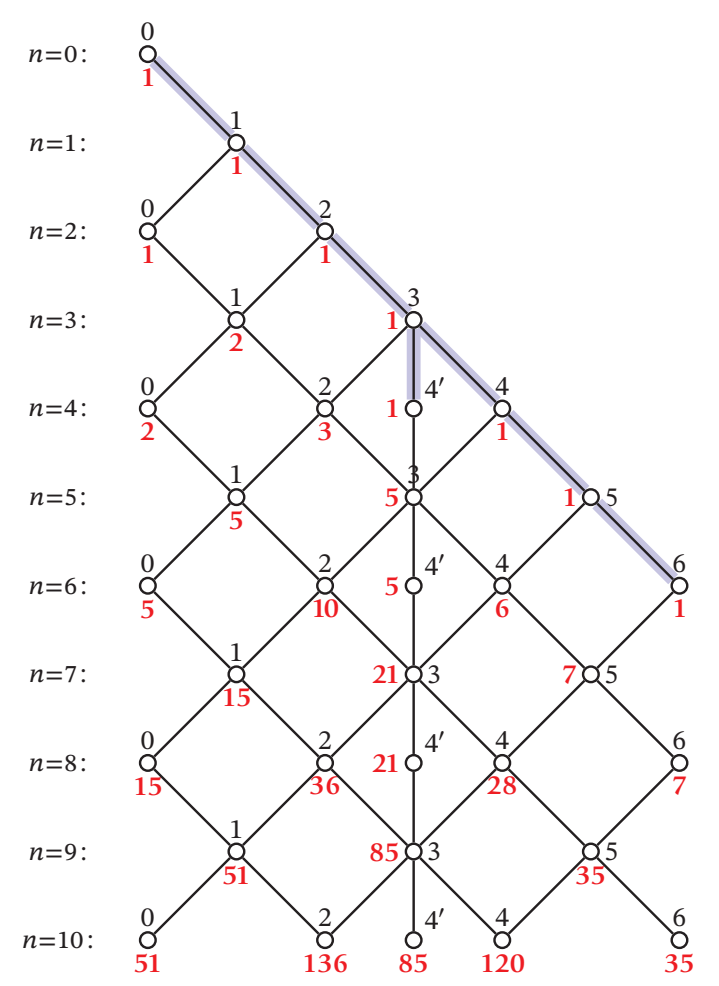

Figure 8. The Bratteli diagram for $\tilde{E}_{7}$. Surprising and beautiful things happen in this diagram. The Dynkin diagram $\hat{E}_{7}$ is embedded at the top of the Bratteli diagram (shaded in blue). The dimension of the irreducible $Z_{n}$-modules are the red labels, which satisfy a Pascal's triangle-like addition rule. The dimension $\operatorname{dim}\left(Z_{n}\right)$ is the number of paths ending at 0 on level $2 n$, i.e., the numbers $1,1,2,5,15,51, \ldots$. Thus the red number at node 0 on level $2 n$ is the sum of the squares of the red numbers on level $n$. For example, $\operatorname{dim}\left(Z_{5}\right)=5^{2}+5^{2}+1^{2}=51$.

groups and quantum groups like

$$
\begin{gathered}
S L_{2}\left(\mathbb{F}_{p}\right), \quad S L_{3}\left(\mathbb{F}_{p}\right), \quad S L_{2}\left(\mathbb{F}_{2^{n}}\right), \quad S L_{2}\left(\mathbb{F}_{p^{2}}\right), \\
\text { and } \quad U_{\xi}\left(\mathfrak{g \mathfrak { I } _ { 2 }}\right),
\end{gathered}
$$

instead of the octahedral group used in Figure 8.

The game is similar to walking on graphs with representations and the McKay correspondence. You start with an empty mixing bowl, choose a small representation, put it in the bowl, and hand it to the next cook. The second cook chooses a small representation to tensor with, and mixes it into the bowl (i.e., calculates the tensor product with what is already there) and hands it on to the next cook in line. This process continues ...., and there's one person at the restaurant (Persi Diaconis) who always wants to know when the food is going to arrive, i.e., how long it takes for all this mixing and cooking to get to the stationary state.

There are several finicky issues that have to be dealt with:

(a) In characteristic $p$, the tensor products don't always decompose as direct sums. (b) Tensoring by the natural module $V$ doesn't always produce all representations.

They fix the issue in (a) by using the Grothendieck ring (Brauer characters) in some cases and by using indecomposable representations instead of irreducible representations in others. They fix the issue in (b) by tensoring by $V \oplus$ triv or by tensoring with $V \oplus V^{(p)}$, where $V^{(p)}$ is the Frobenius twist of $V$ by the $p$ th power field automorphism, and by restricting attention only to the representations of a normal subgroup called the Frobenius kernel.

A few selected answers for the walks and their convergence rates are as follows:

(a) For $G=S L_{2}\left(\mathbb{F}_{p^{2}}\right)$, when mixing (tensoring) by the two-dimensional natural representation $V$ at each step, the walk takes $p^{4}$ steps to equilibriate.

(b) For $G=S L_{2}\left(\mathbb{F}_{2^{n}}\right)$, when walking (tensoring) by the two-dimensional natural representation $V$ at each step, the mixing takes $2^{2 n}$ steps to converge to stationarity.

See Figure 9 and Figure 10.

The bottom line. Georgia Benkart is a clear and creative writer and speaker, who finds great joy in peppering her talks with inventive, mostly deadpan, and always amusing mathematical puns.

The first article Georgia coauthored as an undergraduate appeared in the Pi Mu Epsilon Journal. She was crushed when the publication appeared: they had listed her name as George Benkart. This rather inauspicious beginning to publishing papers was followed by graduate school at Yale University and, in 1974, a postdoc at the University of Wisconsin-Madison. By 1983, she had risen to full professor at Wisconsin-Madison. In 1991, of the 340 tenured or tenure-track faculty at the ten top-ranked schools in mathematics, 12 were women (see Table 2 in [BLW21]). Two decades prior to 1991 , it was likely a quarter to a half that number.

Georgia's research on Lie theory, representation theory, combinatorics, and noncommutative algebra has resulted in over 130 journal publications and research monographs. The more than 350 invited talks she has given during her career include plenary lectures at the Joint Mathematics Meetings on three different occasions and at the annual meetings of the Canadian Mathematical Society and the Mathematical Association of America. In 2014, she was chosen to give both the AWM Noether Lecture at the Joint Mathematics Meetings and the International Mathematical Union's Emmy Noether Lecture at the International Congress of Mathematicians in Seoul.

Georgia was President of the Association for Women in Mathematics from 2009 to 2011 and one of the five US delegates to the 2014 International Mathematical Union General Assembly. She has served as an Associate Secretary of 


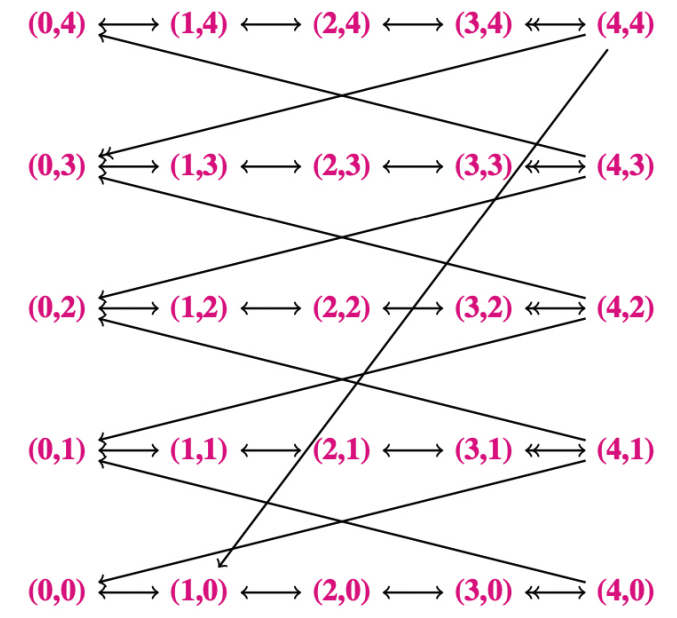

Figure 9. The representation graph for $G=S L_{2}\left(\mathbb{F}_{p^{2}}\right)$ and the two-dimensional natural representation $V=(1,0)$. The double headed arrows indicate that the representation appears twice in the tensor product decomposition. The walk has a drift to the left and a drift downward. Heuristically, the walk moves back and forth at a fixed horizontal level. Once it hits the right-hand wall, it usually bounces back, but with small probability (order $\frac{1}{p}$ ), it jumps up or down by one level. The walk takes order $p^{4}$ steps to totally equilibriate.

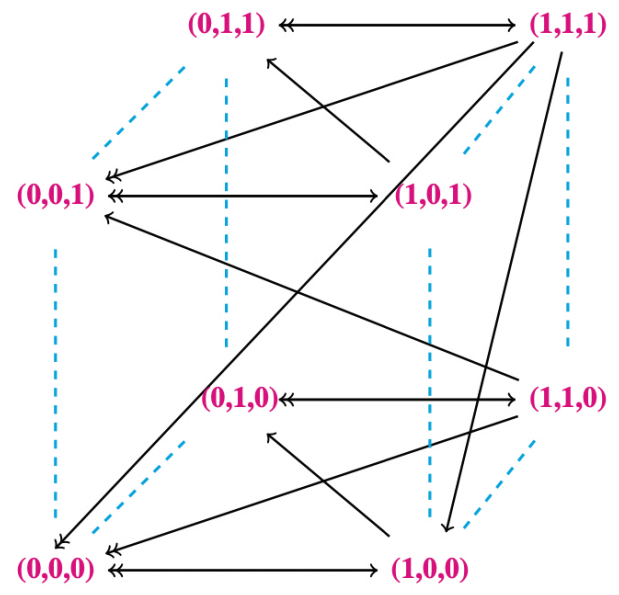

Figure 10. The representation graph for $G=S L_{2}\left(\mathbb{F}_{2^{3}}\right)$ and the two-dimensional natural representation $V=(1,1,0)$. The double headed arrows indicate that the representation appears twice in the tensor product decomposition. For $G=S L_{2}\left(\mathbb{F}_{2^{3}}\right)$, this walk takes order $2^{2 \cdot 3}$ steps to reach stationarity.

the AMS, as a member of the AMS Council, as a member of the US National Committee for Mathematics of the National Academies, and on several editorial boards, including Journal of Algebra, Algebra and Number Theory, and AMS Mathematical Surveys and Monographs.

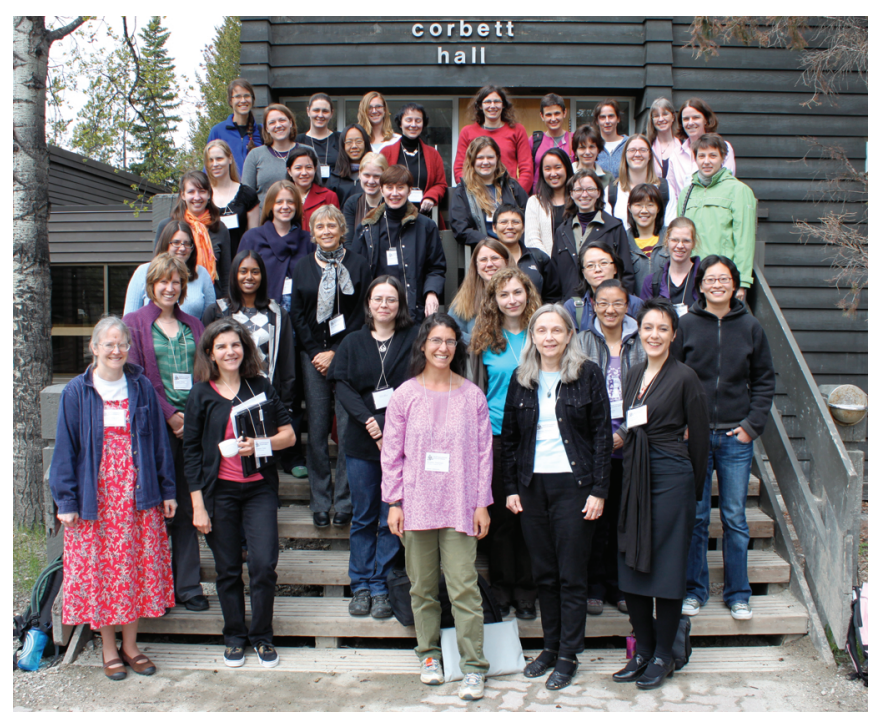

Figure 11. 2011 "Algebraic Combinatorixx (11w5025)" workshop, taken at Banff International Research Station in Banff, Alberta.

However, we feel that Georgia's contribution to our discipline goes well beyond this astonishing catalog of research papers, monographs, lectures, and service roles. She has left an indelible mark on a generation of mathematicians through supportive collaborations with more than 90 coauthors, many of whom are (or, more accurately, were) early-career researchers. And there are even more mathematicians who were not her coauthors but for whom Georgia's mentoring, advice, and support made it possible for them to achieve much more than they ever expected of themselves. Georgia, always humbly and perfectly, serves as a role model and mentor to all.

In the acknowledgments at the opening of her PhD thesis Georgia thanked the many people who supported her by saying,

Many people have contributed to my mathematical education. I owe them all my sincerest thanks.

I would like to express my special appreciation to my advisor, Professor Nathan Jacobson, and to Professor George Seligman who first suggested inner ideals as a possible avenue of research. Among the other individuals who helped with the preparation of this dissertation are Professors Wallace Martindale and James Lepowsky, Darrell Haile, Carl Bumiller, Nicholas Bourbaki, Jr., and Mary Ellen DelVecchio. I am also profoundly grateful for the financial support awarded me through National Science Foundation graduate fellowships and National Science Foundation grant GP-33591.

Now in 2022 it is our turn to sincerely thank Georgia for teaching us so much beautiful mathematics and helping us to begin and sustain careers in research mathematics. Most meaningful to us all is her kindness, decency, and humanity. 


\section{References}

[ABG02] Bruce Allison, Georgia Benkart, and Yun Gao, Lie algebras graded by the root systems $B C_{r}, r \geq 2$, Mem. Amer. Math. Soc. 158 (2002), no. 751, $\mathrm{x}+158$, DOI 10.1090/memo/0751. MR1902499

[BBK ${ }^{+}$21] Georgia Benkart, Rekha Biswal, Ellen Kirkman, Van C. Nguyen, and Jieru Zhu, McKay matrices for finitedimensional Hopf algebras, Canadian Journal of Mathematics (2021), 1-46.

[BBL90] G. M. Benkart, D. J. Britten, and F. W. Lemire, Stability in modules for classical Lie algebras-a constructive approach, Mem. Amer. Math. Soc. 85 (1990), no. 430, vi+165, DOI 10.1090/memo/0430. MR1010997

$\left[\mathrm{BCH}^{+}\right.$94] Georgia Benkart, Manish Chakrabarti, Thomas Halverson, Robert Leduc, Chanyoung Lee, and Jeffrey Stroomer, Tensor product representations of general linear groups and their connections with Brauer algebras, J. Algebra 166 (1994), no. 3, 529-567, DOI 10.1006/jabr.1994.1166. MR1280591

[BDLT20] Georgia Benkart, Persi Diaconis, Martin W. Liebeck, and Pham Huu Tiep, Tensor product Markov chains, J. Algebra 561 (2020), 17-83, DOI 10.1016/j.jalgebra.2019.10.038. MR4135538

[Ben90] Georgia Benkart, Partitions, tableaux, and stability in the representation theory of classical Lie algebras, Lie theory, differential equations and representation theory (Montreal, PQ, 1989), Univ. Montréal, Montreal, QC, 1990, pp. 47-76. MR1121952

[Ben96] Georgia Benkart, Commuting actions-a tale of two groups, Lie algebras and their representations (Seoul, 1995), Contemp. Math., vol. 194, Amer. Math. Soc., Providence, RI, 1996, pp. 1-46, DOI 10.1090/conm/194/02387. MR1395593

[BGP09] Georgia Benkart, Thomas Gregory, and Alexander Premet, The recognition theorem for graded Lie algebras in prime characteristic, Mem. Amer. Math. Soc. 197 (2009), no. 920, xii+145, DOI 10.1090/memo/0920. MR2488391

[BLW21] Georgia Benkart, Kristin Lauter, and Sylvia Wiegand, AWM at 50 and beyond, Notices Amer. Math. Soc. 68 (2021), no. 3, 387-397, DOI 10.1090/noti2239. MR4218176

[BO84] Georgia M. Benkart and J. Marshall Osborn, Rank one Lie algebras, Ann. of Math. (2) 119 (1984), no. 3, 437-463. MR744860

[BO90] Georgia Benkart and J. Marshall Osborn, Simple Lie algebras of characteristic $p$ with dependent roots, Trans. Amer. Math. Soc. 318 (1990), no. 2, 783-807. MR955488

[BR98] Georgia Benkart and Tom Roby, Down-up algebras, J. Algebra 209 (1998), no. 1, 305-344, DOI 10.1006/jabr.1998.7511. MR1652138

[Bri71] E. Brieskorn, Singular elements of semi-simple algebraic groups, Actes du Congrès International des Mathématiciens (Nice, 1970), Gauthier-Villars, Paris, 1971, pp. 279-284. MR0437798

[BZ96] Georgia Benkart and Efim Zelmanov, Lie algebras graded by finite root systems and intersection matrix algebras, Invent. Math. 126 (1996), no. 1, 1-45, DOI 10.1007/s002220050087. MR1408554
[Eld11] Alberto Elduque, Tits construction of the exceptional simple Lie algebras, Pure Appl. Math. Q. 7 (2011), no. 3, Special Issue: In honor of Jacques Tits, 559-586, DOI 10.4310/PAMQ.2011.v7.n3.a4. MR2848587

[Kac90] Victor G. Kac, Infinite-dimensional Lie algebras, 3rd ed., Cambridge University Press, Cambridge, 1990, DOI 10.1017/CBO9780511626234, MR1104219

[KŠ66] A. I. Kostrikin and I. R. Šafarevič, Cartan's pseudogroups and the p-algebras of Lie (Russian), Dokl. Akad. Nauk SSSR 168 (1966), 740-742. MR0199235

[Sel67] G. B. Seligman, Modular Lie algebras, Ergebnisse der Mathematik und ihrer Grenzgebiete, Band 40, SpringerVerlag New York, Inc., New York, 1967. MR0245627

[Str13] Helmut Strade, Simple Lie algebras over fields of positive characteristic. III: Completion of the classification, De Gruyter Expositions in Mathematics, vol. 57, Walter de Gruyter GmbH \& Co. KG, Berlin, 2013. MR3025870

[Str17a] Helmut Strade, Simple Lie algebras over fields of positive characteristic. Vol. 1: Structure theory, De Gruyter Expositions in Mathematics, vol. 38, De Gruyter, Berlin, 2017. Second edition [of MR2059133]. MR3642321

[Str17b] Helmut Strade, Simple Lie algebras over fields of positive characteristic. Vol. II: Classifying the absolute toral rank two case, De Gruyter Expositions in Mathematics, vol. 42, De Gruyter, Berlin, 2017. Second edition [of MR2573283]. MR3642323

[Sun90] Sheila Sundaram, Tableaux in the representation theory of the classical Lie groups, Invariant theory and tableaux (Minneapolis, MN, 1988), IMA Vol. Math. Appl., vol. 19, Springer, New York, 1990, pp. 191-225. MR1035496

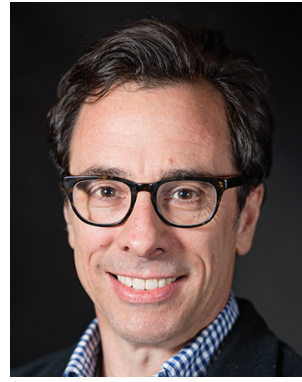

Tom Halverson

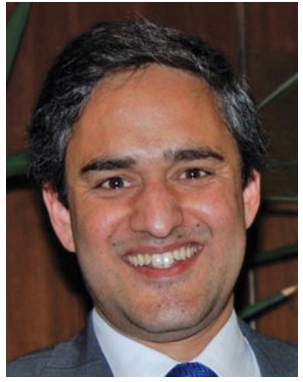

Arun Ram
Credits

Opening photo is courtesy of Yvonne Nagel.

Figures 1 and 3-8 are courtesy of Tom Halverson.

Figure 2 is courtesy of the University of Texas.

Figures 9 and 10 are courtesy of Georgia Benkart. Graphics appeared previously in Georgia Benkart, Persi Diaconis, Martin W. Liebeck, and Pham Huu Tiep, "Tensor product Markov chains," Journal of Algebra, Volume 561, 2020.

Figure 11 is courtesy of the Banff International Research Station.

Photo of Tom Halverson is courtesy of David J. Turner / Macalester College.

Photo of Arun Ram is courtesy of Yvonne Nagel. 